\title{
A PROBABILISTIC GENERALIZATION OF MATRIC BANACH ALGEBRAS
}

\author{
B. O. KOOPMAN
}

1. Introduction. One of the simplest examples of a noncommutative Banach algebra ${ }^{1}$ is provided by the class of all $r$-rowed square matrices whose elements belong to the complex (or else the real) field. Matrix addition, multiplication, and scalar multiplication by numbers in the corresponding field being defined as usual, any one of several familiar norms $N$ may be introduced. It is convenient for the objectives of this note to write, following E. Hille, ${ }^{2}$

$$
N\left(\left\|a_{i j}\right\|\right)=\max _{i=1, \ldots, r} \sum_{j=1}^{r}\left|a_{i j}\right| .
$$

The generalization considered here consists essentially in regarding each subscript $i$ and $j$ in the matrix elements $a_{i j}$ as ranging over the elements of a fixed, absolutely general, abstract set $X$ (or "space"but without assumptions of topology). Summation over subscripts is replaced by general abstract integration over $X$; and a direct extension of the norm $N\left(\left\|a_{i j}\right\|\right)$ just defined is given in terms of absolute variations of additive set functions.

In addition to the set $X$ (which, we assume, has at least one element), we consider a fixed class $X$ of subsets of $X$ forming a $\sigma$-algebra: it contains the null set $O$ as well as $X$ itself; and it is closed with respect to countable union $(\cup)$, intersection $(\cap)$, and complementation $\left(E^{\prime}=X-E\right)$, and therefore with respect to the formation of set limits. $^{3}$

Finally, we introduce the aggregate $\mathfrak{B}$ of all complex-valued functions $\phi=\phi(x, E)$ having the four following properties:

(i) $\phi(x, E)$ is defined for all $x \in X$ and $E \in X$;

(ii) $|\phi(x, E)| \leqq M_{\phi<\infty}$ for all $x \in X$ and $E \in X$;

(iii) for each fixed $x \in X, \phi(x, E)$ is a countably additive function of the set $E$;

(iv) for each fixed $E \in X, \phi(x, E)$ is a measurable $(X)$ function of $x$.

Presented to the Society, June 17, 1950; received by the editors June 5, 1950.

${ }^{1}$ For the notions involved in a Banach algebra, cf., for example, E. Hille, Functional analysis and semi-groups, Amer. Math. Soc. Colloquium Publications, vol. 31, New York, 1948, \$1.14.

${ }^{2}$ E. Hille, loc. cit., \$21.7.

${ }^{3}$ Cf. for example, P. R. Halmos, Measure theory, New York, van Nostrand, 1950, $\S 6(4)$. The terminology of this book will be used throughout the present note. 
The elements of $\mathscr{B}$ play the role of the matrices in the finite case: after defining their algebraic combinations and norms, it will be shown that they form a Banach algebra.

Our study is oriented toward probability: The elements $x$ of $X$ are (or correspond to) the possible outcomes of a random trial of a given kind. The sets $E$ of $X$ represent the "experimentally observable" events - the occurrence of $E$ meaning that the outcome $x$ of the trial falls in $E$. Then, in addition to the probabilities $p(E)$ of such events, it is necessary to consider the conditional probabilities $p(x, E)$ of the event $E$ of a stated trial when another stated trial is assumed to have the outcome $x$. Probability reasoning naturally leads one to assume properties (i)-(iv) of $\phi(x, E)=p(x, E)$. But since it is necessary to form linear combinations with complex coefficients of such functions and then to take their limits (for example, in expected values and generating functions), one is compelled to envisage the general class $\mathfrak{B}$.

The results of this note have applications to one-stop Markoff chains of very general types: $:^{4}$ the expressions $p(x, E)$ are the "transition probabilities" $x \rightarrow E$.

2. The absolute variation. It is necessary to recall certain properties of the absolute (or total) variation of complex-valued set functions. Let $\mu=\mu(E)$ be such a function, defined for all $E \in X$, bounded over $X$, and countably additive. Then its absolute variation $\mu^{v}(E)$ is defined as ${ }^{5}$

$$
\mu^{\nabla}(E)=\sup _{f}\left|\int_{E} f d \mu\right|,
$$

where $f=f(x)$ ranges over the class of all complex-valued measurable $(X)$ functions of $x \in X$ having $|f(x)| \leqq 1$.

That $\mu^{\nabla}(E)$ is bounded over $X$, and, indeed, that it satisfies the inequality

$$
0 \leqq \mu^{V}(E) \leqq 8 M_{\mu}, \quad M_{\mu}=\sup _{E \in \mathcal{X}}|\mu(E)|,
$$

is seen as follows: Splitting into real imaginary parts $\mu=\mu_{1}+i \mu_{2}$, $f=f_{1}+i f_{2}$, we have

$$
\int_{E} f d \mu=\int_{E} f_{1} d \mu_{1}-\int_{E} f_{2} d \mu_{2}+i \int_{E} f_{2} d \mu_{1}+i \int_{E} f_{1} d \mu_{2}
$$

\footnotetext{
${ }^{4}$ Special cases of our Banach algebra have been used in this connection; cf., for example, E. Hille, loc. cit., $\$ 21.10$.

$\checkmark$ Cf. P. R. Halmos, loc. cit., $\$ 29$ (8).
} 
Now let $X=A_{k} \cup B_{k}\left(A_{k} \cap B_{k}=0\right)$ be a Hahn decomposition ${ }^{6}$ of $X$ corresponding to the real set function $\mu_{k}(k=1,2), \mu_{k}(F) \geqq 0$ for all $F \subset A_{k}$ and $\mu_{k}(F) \leqq 0$ for all $F \subset B_{k}$. Then, by the elements of abstract integration, we obtain (for $j=1,2$ )

$$
\left|\int_{E} f_{j} d \mu_{k}\right| \leqq \sup _{x \in E}\left|f_{j}(x)\right|\left[\mu_{k}\left(E \cap A_{k}\right)-\mu_{k}\left(E \cap B_{k}\right)\right] \leqq 2 M_{\mu}
$$

(since $\left|f_{j}\right| \leqq|f| \leqq 1$ and $\left|\mu_{k}\right| \leqq|\mu| \leqq M_{\mu}$ ). Combining this with the preceding equation, and applying (2.1), the relation (2.2) is established.

When $\mu$ is real-valued, $\mu^{V}(E)$ reduces to the usual form of the absolute variation, namely, $\mu(E \cap A)-\mu(E \cap B)$, where $X=A \cup B$ $(A \cap B=0)$ is a Hahn decomposition of $X$ for $\mu$. For on the one hand, taking $f(x)=\chi_{A}(x)-\chi_{B}(x)$ (the $\chi$ 's being the characteristic functions of $A, B)$, we have

$$
\begin{aligned}
\int_{E} f d \mu & =\int_{E \cap_{A}} f d \mu+\int_{E \cap_{B}} f d \mu \\
& =\int_{E \cap_{A}} d \mu-\int_{E \cap_{B}} d \mu=\mu(E \cap A)-\mu(E \cap B),
\end{aligned}
$$

so that $\mu^{v}(E) \geqq \mu(E \cap A)-\mu(E \cap B)$. On the other hand, this $(\geqq)$ is replaceable by $(\leqq)$. Let $f(x)$ be any function, measurable $(X)$ and with $|f(x)| \leqq 1$. That $\int_{E} f d \mu$ is finite results from (2.2); and the case $\int_{E} f d \mu=0$ requires no proof. In all other cases, let $\omega$ be the (smallest non-negative) argument of $\int_{E} f d \mu$, and write

$$
g(x)=e^{-i \omega} f(x), \quad g(x)=g_{1}(x)+i g_{2}(x),
$$

$\left(g_{1}, g_{2}\right.$, real $)$. Clearly

$$
\begin{aligned}
\left|\int_{E} f d \mu\right| & =\int_{E} g d \mu=\int_{E} g_{1} d \mu+i \int_{E} g_{2} d \mu=\int_{E} g_{1} d \mu \\
& \leqq \sup _{x \in E}\left|g_{1}(x)\right|[\mu(E \cap A)-\mu(E \cap B)]
\end{aligned}
$$

the latter by elementary real integration. Since $\left|g_{1}(x)\right| \leqq|g(x)| \leqq 1$, the desired inequality is obtained, thus completing the proof.

The following property is an immediate consequence of (2.1):

$$
\left|\int_{E} f d \mu\right| \leqq \mu^{V}(E) \sup _{x \in E}|f(x)|, \quad \text { for any } f(x) \text { measurable }(\mathcal{X}) \text {. }
$$

' Cf. P. R. Halmos, loc. cit., \$29. 
It is trivial when $f(x)$ is unbounded or identically zero, and is proved in the bounded case by applying (2.1) with the previous $f(x)$ replaced by $f(x) /$ sup $|f(x)|$.

A further property results from an obvious application of the definition (2.1) to two set functions of the present type:

$$
(a \mu+b \nu)^{v}(E) \leqq|a| \mu^{v}(E)+|b| \nu^{v}(E) .
$$

Here $a$ and $b$ are complex constants and the superscript $V$ always denotes the absolute variation.

A less immediate property is that $\mu^{V}$ is countably additive over $X$. For any sequence $\left\{E_{n}\right\}$ with

$$
E=\cup_{n} E_{n}, \quad E_{i} \cap E_{j}=0 \quad(i \neq j), \quad E_{n} \in \mathcal{X}
$$

we shall have

$$
\mu^{V}(E)=\sum_{n} \mu^{V}\left(E_{n}\right) .
$$

The fact that $\mu^{V}(E) \leqq \sum_{n} \mu^{V}\left(E_{n}\right)$ follows from (2.1) and from the relations

$$
\left|\int_{E} f d \mu\right|=\left|\sum_{n} \int_{E_{n}} f d \mu\right| \leqq \sum_{n}\left|\int_{E_{n}} f d \mu\right| .
$$

Hence if (2.6) is false, we should have a finite constant $h$ and

$$
\sum_{n} \mu^{V}\left(E_{n}\right)-\mu^{V}(E) \geqq h>0 .
$$

(Thus use of $\geqq h$ instead of $=h$ is to allow for the possibility of a divergent sum on the left.) There would then exist a sequence $\left\{f_{n}\right\}$ of functions (measurable $(X),\left|f_{n}(x)\right| \leqq 1$ ) such that

$$
\left|\int_{E_{n}} f_{n} d \mu\right| \geqq \mu^{V}\left(E_{n}\right)-\frac{h}{2^{n+1}} .
$$

Consequently

$$
\mu^{V}(E)<\sum_{n}\left|\int_{E_{n}} f_{n} d \mu\right| .
$$

Dropping any terms on the right that are zero, let $\omega_{n}$ be the (smallest non-negative) argument of $\int_{E_{n}} f_{n} d \mu$. Set

$$
g(x)=e^{-i \omega_{n}} f_{n}(x) \quad \text { for } \quad x \in E_{n} \quad(n=1,2, \cdots) .
$$

Then clearly $|g(x)| \leqq 1$ and $g(x)$ is measurable $(X)$. Furthermore, the preceding sum may be written in terms of $g(x)$ without absolute value signs. Therefore, finally, 


$$
\mu^{V}(E)<\sum_{n} \int_{E_{n}} g d \mu=\int_{E} g d \mu,
$$

contrary to the definition of $\mu^{V}(E)$. Thus (2.5) is proved.

In view of the additivity and non-negativeness of $\mu^{v}$, we see at once that $E \subset F$ implies $\mu^{v}(E) \subset \mu^{v}(F)$. This, in connection with (2.1) and (2.2), shows that

$$
0 \leqq \mu^{V}(X)<\infty ; \quad \mu^{V}(X)=0 \text { if and only if } \mu(E) \equiv 0 .
$$

The following related property, applying to any sequence $\left\{\mu_{n}\right\}$ of set functions of type $\mu$, is almost as evident:

$$
\underset{\mu_{n}}{V}(X) \rightarrow 0 \quad \text { if and only if } \mu_{n}(E) \rightarrow 0 \text { uniformly over } X .
$$

This follows from the inequality

$$
\left|\mu_{n}(E)\right|=\left|\int_{X} \chi_{E} d \mu_{n}\right| \leqq \mu_{n}^{V}(X) \leqq 8 M_{\mu_{n}},
$$

(cf. (2.1), (2.2)), where $X_{E}$ is the characteristic function of $E$; and from the fact that $\mu_{n}(E) \rightarrow 0$ uniformly over $X$ if and only if $M_{\mu_{n}} \rightarrow 0$.

Relations (2.4) and (2.6) show that $\mu^{V}(X)$ has the properties of a norm in the space of all $\mu$ of the present type; and (2.7) easily leads to the completeness of this space under this norm. Therefore the space is a Banach space, as is known. The Banach algebra $\mathfrak{B}$ which we seek is a related but more general structure.

3. The algebraic properties in $\mathfrak{B}$. Returning to the aggregate $\mathfrak{B}$ of functions $\phi=\phi(x, E)$ having the properties (i)-(iv) given in $\$ 1$, we shall define addition, scalar multiplication, and multiplication of pairs of elements. The first two are defined at once by setting, for any $\alpha, \beta \in \mathfrak{B}$, and complex constants $a, b$,

$$
\gamma=a \alpha+b \beta=a \alpha(x, E)+b \beta(x, E)=\gamma(x, E) .
$$

Evidently $\gamma \in \mathfrak{B}$. Therefore $\mathfrak{B}$ is a linear system $^{7}$ over the complex field, and its "zero" is $\theta=\theta(x, E) \equiv 0$.

Next, the (noncommutative) product $\alpha \beta$ of two elements $\alpha, \beta \in \mathfrak{B}$ is defined as the abstract integral over $X$ of $\beta$ (regarded as a function of its first variable) with respect to the complex measure $\alpha$ (regarded as a set function of its second variable). This is conveniently expressed by the following slightly modified symbolism for integration:

$$
\gamma=\alpha \beta=\int_{X} \alpha(x, d y) \beta(y, E)=\gamma(x, E) .
$$

\footnotetext{
${ }^{7}$ Cf. E. Hille, loc. cit., $\$ 1.9$.
} 
Here, $y$ is a "variable of integration," while $x$ and $E$ remain fixed in the process.

We must first prove that this $\gamma \in \mathfrak{B}$. Property (i) results from the general theory of integration. The integral in (3.2) exists for each $(x, E)$, since $\beta(y)=\beta(y, E)$ is bounded and measurable $(X)$, and $\alpha(F)$ $=\alpha(x, F)$ is countably additive and of bounded variation $\alpha^{V}(X)$. Property (ii) follows from (2.2) and (2.3), together with the validity of (ii) for $\alpha$ and $\beta$.

$$
\begin{aligned}
|\gamma(x, E)| & =\left|\int_{X} \alpha(x, d y) \beta(y, E)\right| \leqq \alpha^{V}(x, X) \sup _{y \in X}|\beta(y, E)| \\
& \leqq 8 M_{\alpha} M_{\beta} .
\end{aligned}
$$

To establish (iii), let $\left\{E_{n}\right\}$ be a sequence of disjoint sets in $X$. The application of Lebesgue's Bounded Convergence Theorem ${ }^{8}$ gives the desired result:

$$
\begin{aligned}
\gamma\left(x, \cup_{n} E\right) & =\int_{X} \alpha(x, d y) \beta\left(y, \cup_{n} E_{n}\right) \\
& =\int_{X} \alpha(x, d y) \sum_{n} \beta\left(y, E_{n}\right) \\
& =\sum_{n} \int_{X} \alpha(x, d y) \beta\left(y, E_{n}\right) \\
& =\sum_{n} \gamma\left(x, E_{n}\right) .
\end{aligned}
$$

Property (iv) is shown in two steps. Fixing $E$, write $\beta(y)=\beta(y, E)$. Suppose first that $\beta(y)$ is simple: on the class $\left\{C_{1}, \cdots, C_{n}\right\}$ of sets of $X$ such that

$$
C_{1} \cup \cdots \cup C_{n}=X, \quad C_{i} \cap C_{j}=0 \quad(i \neq j) .
$$

We have

$$
\beta(y)=c_{i} \text { when } y \in C_{i}(i=1, \cdots, n) .
$$

Then

$$
\int_{X} \alpha(x, d y) \beta(y)=\sum_{i=1}^{n} c_{i} \alpha\left(x, C_{i}\right)
$$

and this is measurable $(X)$, being a linear combination of functions measurable $(X)$. In the general case, represent $\beta(y)$ as the limit of a sequence of simple functions

${ }^{8}$ Cf. P. R. Halmos, loc. cit., §26(D). 


$$
\beta(y)=\lim _{n \rightarrow \infty} \beta_{n}(y)
$$

(this possibility in the complex case results from the conventional result for reals, by splitting into real and imaginary parts). Then by Lebesgue's bounded convergence theorem,

$$
\begin{aligned}
\gamma(x, E) & =\int_{X} \alpha(x, d y) \lim _{n \rightarrow \infty} \beta_{n}(y) \\
& =\lim _{n \rightarrow \infty} \int_{X} \alpha(x, d y) \beta_{n}(y),
\end{aligned}
$$

so that $\gamma(x, E)$, being the limit of functions just shown to be measurable $(X)$, is itself measurable $(X)$.

This completes the proof that $\gamma=\alpha \beta \in \mathfrak{B}$.

Having established the closure of $\mathfrak{B}$ under addition, multiplication, and scalar multiplication, we shall show that $\mathfrak{B}$ contains the identical element $\epsilon$. Define $\epsilon$ as follows:

$$
\epsilon=\epsilon(x, E)=\left\{\begin{array}{l}
1 \text { when } x \in E, \\
0 \text { when } x \notin E .
\end{array}\right.
$$

In other words, $\epsilon=\chi_{E}(x)$, the characteristic function of $E$. We must show that $\phi \epsilon=\phi$ and $\epsilon \phi=\phi$.

The first is immediate, since

$$
(\phi \epsilon)(x, E)=\int_{X} \phi(x, d y) \epsilon(y, E)=\int_{X} \phi(x, d y) \chi_{E}(y)=\phi(x, E) .
$$

To prove the second, let $x, E$, and a constant $h>0$ be given, and let $H$ be the set of points $y$ of $X$ for which

$$
H:|\phi(y, E)-\phi(x, E)|<h .
$$

Evidently $H \in X$ and $x \in H$, and we have

$$
(\epsilon \phi)(x, E)=\left(\int_{H}+\int_{H^{\prime}}\right) \epsilon(x, d y) \phi(y, E) .
$$

But the $\int_{H^{\prime}}$ is zero: on the one hand, by (2.3) and (ii), it is majorized by $\epsilon^{V}\left(x, H^{\prime}\right) M_{\phi}$; and on the other hand, $\epsilon^{V}\left(x, H^{\prime}\right)=\epsilon\left(x, H^{\prime}\right)=0$, since $\epsilon \geqq 0$ and $x \notin H^{\prime}$. Therefore

$$
(\epsilon \phi)(x, E)=\int_{H} \epsilon(x, d y) \phi(y, E) .
$$

But we may evidently write 


$$
\phi(x, E)=\int_{H} \epsilon(x, d y) \phi(x, E) .
$$

Consequently

$$
\begin{aligned}
|(\epsilon \phi)(x, E)-\phi(x, E)| & =\int_{H} \epsilon(x, d y)|\phi(y, E)-\phi(x, E)| \\
& \leqq \epsilon^{V}(x, H) h=h,
\end{aligned}
$$

and this being true for all $h>0, \epsilon \phi=\phi$ follows.

It remains to show that our linear system, with multiplication and the identity, is actually a linear algebra (ring with scalar multiplication); that is, we must establish the relationship

$$
(a \alpha+b \beta) \gamma=a(\alpha \gamma)+b(\beta \gamma), \quad \gamma(a \alpha+b \beta)=a(\gamma \alpha)+b(\gamma \beta),
$$

between multiplication and the linear combinations; and must prove the associativity of multiplication, $a(\beta \gamma)=(\alpha \beta) \gamma$. The former is an immediate consequence of (3.1), (3.2), and the elementary properties of integrals.

The proof of associativity is equivalent to the proof of the following equation, which asserts the independence of the order of integration in an iterated integral (we indicate the variables of integration by superscripts):

$$
\begin{aligned}
\alpha(\beta \gamma) & =\int_{X}^{y} \alpha(x, d y)\left[\int_{X}^{z} \beta(y, d z) \gamma(z, E)\right] \\
& =\int_{X}^{z}\left[\int_{X}^{y} \alpha(x, d y) \beta(y, d z)\right] \gamma(z, E)=(\alpha \beta) \gamma .
\end{aligned}
$$

In these integrations, $x$ and $E$ remain fixed. Write $\gamma(x)=\gamma(z, E)$. The equation is evident when $\gamma(z)$ is a simple function: an obvious computation gives

$$
\alpha(\beta \gamma)=\sum_{k=1}^{n} c_{k} \int_{X}^{\nu} \alpha(x, d y) \beta\left(y, C_{k}\right)=(\alpha \beta) \gamma
$$

$\left(c_{k}, C_{k}\right.$ being related to $\gamma$ as they were to $\beta$ in the earlier use of simple functions). In the general case, by taking a sequence $\left\{\gamma_{n}(x)\right\}$ of simple functions with $\gamma_{n} \rightarrow \gamma$, the application of Lebesgue's bounded convergence theorem shows that (for the particular $x, E$ considered)

$$
\begin{aligned}
& \alpha(\beta \gamma)=\alpha\left(\beta \lim \gamma_{n}\right)=\alpha\left(\lim \beta \gamma_{n}\right)=\lim \alpha\left(\beta \gamma_{n}\right), \\
& (\alpha \beta) \gamma=(\alpha \beta) \lim \gamma_{n}=\lim (\alpha \beta) \gamma_{n}
\end{aligned}
$$


and since, as we have just shown, $\alpha\left(\beta \gamma_{n}\right)=(\alpha \beta) \gamma_{n}$, the desired property $(\alpha \beta) \gamma=\alpha(\beta \gamma)$ follows.

4. The norm in $\mathfrak{B}$. We now introduce the norm $N(\phi)$ for every element $\phi$ of $\mathfrak{B}$; it is clearly the direct extension to $\mathfrak{B}$ of the matric norm of (1.1).

$$
N(\phi)=\sup _{x \in X} \phi^{V}(x, X) .
$$

In view of (2.1), this is equivalent to writing

$$
N(\phi)=\sup _{x \in X ;|f| \leqq 1}\left|\int_{X} \phi(x, d y) f(y)\right| .
$$

The following properties are evident consequences of (4.1), (ii), and the properties established in $\S 2$.

(4.3) $\quad N(c \phi)=|c| N(\phi)$ $N(\phi)=0$ if and only if $\phi=\theta$ (the “zero").

(4.4) $\quad N(\alpha+\beta) \leqq N(\alpha)+N(\beta)$.

And as a consequence of (3.3), and so on, we have immediately

$$
N(\epsilon)=1 \text {. }
$$

The next property is that

$$
N(\alpha \beta) \leqq N(\alpha) N(\beta) .
$$

For the proof, introduce $\gamma(x, E)$ as in (3.2) and let $f(x)$ be any measurable $(X)$ function with $|f(x)| \leqq 1$. Then, if we indicate the variable of integration by a superscript, we have

$$
\begin{aligned}
\int_{X}^{z} \gamma(x, d z) f(z) & =\int_{X}^{z}\left[\int_{X}^{y} \alpha(x, d y) \beta(y, d z)\right] f(z) \\
& =\int_{X}^{y} \alpha(x, d y)\left[\int_{X}^{z} \beta(y, d z) f(z)\right] .
\end{aligned}
$$

The last equation is readily proved by the method used at the end of $\$ 3$ in the proof of associativity: Direct computation establishes the equation when $f(z)$ is a simple function; Lebesgue's bounded convergence theorem extends the result so obtained to the general case. It may be noted that $f(z)$ is so far merely assumed bounded and measurable $(\mathcal{X})$.

We now restrict $f(z)$ to satisfy $|f(x)| \leqq 1$. Then by the equation just established, in conjunction with (2.3), and so on, 


$$
\begin{aligned}
\left|\int_{X}^{z} \gamma(x, d z) f(z)\right| & =\left|\int_{X}^{y} \alpha(x, d y)\left[\int_{X}^{z} \beta(y, d z) f(z)\right]\right| \\
& \leqq \alpha^{V}(x, X) \sup _{y}\left|\int_{X}^{z} \beta(y, d z) f(z)\right| \\
& \leqq \alpha^{V}(x, X) \sup _{y ;|f| \leqq 1}\left|\int_{X}^{z} \beta(y, d z) f(z)\right| \\
& =\alpha^{V}(x, X) N(\beta),
\end{aligned}
$$

from which (4.6) is an immediate consequence.

Our last task is to examine the topology induced in $\mathfrak{B}$ by the norm $N$. We shall prove the two following theorems for any sequence $\left\{\phi_{n}\right\}, \phi_{n} \in \mathfrak{B}:$

(4.7) $N\left(\phi_{n}\right) \rightarrow 0$ if and only if $\phi_{n}(x, E) \rightarrow 0$ uniformly $(x, E) \in(X, X)$,

(4.8) $N\left(\phi_{n}-\phi_{m}\right) \rightarrow 0$ if and only if $\exists \phi \in \mathfrak{B} \ni N\left(\phi_{n}-\phi\right) \rightarrow 0$.

The first interprets convergence in norm. The second asserts the completeness of $\mathfrak{B}$; when it is proved, the fact that $\mathfrak{B}$ is a Banach algebra with identity will become evident.

The proof of (4.7) parallels that of (2.7) exactly: one has but to write $(2.8)$ in the form

$$
\left|\phi_{n}(x, E)\right|=\left|\int_{X} \phi_{n}(x, d y) \chi_{E}(y)\right| \leqq \phi_{n}^{V}(x, X) \leqq 8 M_{\phi_{n}(x, E)}
$$

and derive from it

$$
\left|\phi_{n}(x, E)\right| \leqq \sup _{x \in X} \phi_{n}^{v}(x, X)=N\left(\phi_{n}\right) \leqq 8 \sup _{x \in E} M_{\phi_{n}(x, E)}=8 M_{\phi_{n}} ;
$$

then one uses the fact that $\phi_{n}(x, E) \rightarrow 0$ uniformly over $x \in X, E \in X$, if and only if $M_{\phi_{n}} \rightarrow 0$.

To prove the completeness, let $N\left(\phi_{n}-\phi_{n}\right) \rightarrow 0$. Then the most obvious extension of the proof of (4.7) shows that $\phi_{n}(x, E)-\phi_{m}(x, E)$ $\rightarrow 0$ uniformly over $(X, X)$. It follows that $\phi_{n}(x, E) \rightarrow \phi(x, E)$ uniformly. By the uniform convergence, this $\phi=\phi(x, E)$ evidently satisfies (i)-(iv): $\phi \in \mathfrak{B}$. Then (4.7) shows that $N\left(\phi_{n}-\phi\right) \rightarrow 0$; that is, $\phi_{n} \rightarrow \phi$ in norm.

Columbia University 